\title{
La Lectura crítica mediada por las TIC en el contexto educativo
}

Artículo de reflexión

Fecha de Recepción: 11 septiembre 2017.

Fecha de Aprobación: 26 febrero 2018.

\section{Resumen}

Éste documento, busca presentar resultados parcialesdeunainvestigación, con el objetivo de establecer una estrategia didáctica basada en el uso de las TIC para fortalecer la lectura crítica en las estudiantes del grado décimo del Colegio Nacionalizado la Presentación en las áreas de Física y Sociales. El marco teórico considera: TIC, estrategia didáctica y lectura crítica. La metodología es cualitativa, el tipo de investigación acción educativa, y se desarrolló en tres etapas. Planificación, mediante recolección de información a partir de observación participante, prueba de habilidades de lectura crítica y revisión documental, con el objetivo de hallar las debilidades en la comprensión lectora. La etapa de acción tuvo como propósito diseñar la

estrategia apoyada en actividades con el grupo sustentadas en las TIC, lo que llevo a la conclusión de que ésta metodología proporciona al estudiante un escenario motivante permitiendo una verdadera interacción autor-lector, haciendo de este ejercicio pedagógico una herramienta que permite analizar, asociar, comparar, proponer, opinar de acuerdo al propio juicio. En la última etapa observación y reflexión se evaluarán resultados en base a la triangulación de la información. y se espera percibir un progreso en el reconocimiento de contenidos valorativos de un texto, desde la lectura crítica.

Palabras clave: Iinvestigación sobre la educación, Lectura de pruebas, técnica de aprendizaje, estrategia de enseñanza.
* Colegio Nacionalizado la Presentación - Boyacá Colombia

alejitasm@gmail.com

$* *$ Colegio Nacionalizado

la Presentación - Boyacá -

Colombia

franseal74@hotmail.com

**** Universidad Pedagógica

y Tecnológica de Colombia

- Boyacá - Colombia

nelsonaugusto.medina@

uptc.edu.co

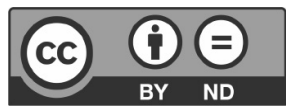




\section{Introducción}

El presenteartículodeinvestigación hace parte de la investigación intitulada Las TIC, como estrategia de mejoramiento para la lectura crítica en física y sociales, en el marco de la Maestría en Educación énfasis Profundización de la Universidad Pedagógica y Tecnológica de Colombia. Éste proyecto se ha venido desarrollando en el Colegio Nacionalizado la Presentación del municipio de Duitama - Boyacá, desde las áreas de Física y Sociales.

En el proceso educativo la lectura además de una actividad lingüística, cognitiva y comunicativa, es una práctica social y cultural que debe promoverse en sus diferentes niveles, por ello desde las aulas se ha venido implementando diferentes proyectos como lectores competentes y plan lector 2017.

No obstante, con base en el análisis del índice sintético de Calidad ISCE 2016, se evidenció un bajo desempeño en la lectura crítica, lo que implica el análisis de la relación explicita e implícita sobre las intenciones del autor del texto y la relación que tiene con la experiencia y dinámica del lector con el texto.

Partiendo de lo anterior, se establece la necesidad de mejorar la lectura crítica articulando nuevos escenarios para posibilitar a los estudiantes utilizar herramientas del entorno escolar, de allí la importancia de involucrar las Tecnologías de la Información y Comunicación en los procesos de enseñanza-aprendizaje como parte de la construcción del conocimiento cuando en la práctica pedagógica docente no se presenta como un poseedor del saber sino como mediador del mismo, lo cual lleva a pensar: ¿Qué aportes puede generar la aplicación de una estrategia didáctica, fundamentada en el uso de las TIC, para mejorar la lectura crítica en las estudiantes de grado 10 ?.

A partir del surgimiento de este interrogante, se planteó como propósito establecer una estrategia didáctica basada en el uso de las TIC para fortalecer la lectura crítica en las estudiantes del grado décimo y posibilitar la construcción de procesos de análisis e interpretación, lo que permite una postura externa del lector frente al texto.

Cabe resaltar que la investigación se encuentra en construcción, por ende, se presentan aspectos de planeación, revisión bibliográfica y estructuración metodológica, con miras a la materialización del proceso.

\section{Una mirada a los antecedentes investigativos en lectura critica}

En este aparte se encuentran investigaciones relacionadas con el uso de las TIC para mejorar la lectura crítica, a través de una descripción seleccionada intencionalmente para documentar el estado actual del tema abordado.

En el artículo de Ortiz (2004), titulado "Lectura y escritura en la era digital", resalta como la aparición de las TIC trae consigo miedos y desafíos en la labor docente. La investigación se 
basó en la comparación de cómo se trasformó el acceso de la información del campo laboral hacia el ámbito escolar, resaltando el impacto en cuanto a la adaptación para estar al avante de la era digital en el quehacer pedagógico, donde la información está al alcance de un clic, exigiendo tanto al docente como al estudiante habilidades de lectura, presentada en diferentes escenarios interactivos que requieren una respuesta en la apropiación y dominio de los nuevos medios. De esta manera el docente adquiere un rol dinámico para no seguir lecciones repetitivas, sino abrir infinidad de posibilidades con espacios interactivos, sincrónicos y asincrónicos de intercambio de información. Por tanto, el estudio permitió concluir que la tecnología se puede convertir en un gran aliado para desarrollar competencias de lectura, y que es responsabilidad de los docentes hacer las adaptaciones para lograr este propósito.

En la investigación de Fainholc (2006) en Argentina titulada "la lectura crítica en internet: desarrollo de habilidades y metodología para su práctica”, donde tuvo como objetivo establecer la relación entre el lector y la información digital en la generación de las competencias lectoras. Las reflexiones que surgieron del estudio resaltaron la importancia de la motivación y el papel que deben jugar en los procesos educativos para la formación de lectores críticos al acceder a la información que lo rodea cotidianamente en internet. Ser un usuario crítico de internet o alcanzar el nivel hiper-lector establece la importancia de definir el acceso a la red no como un medio de comunicación, sino un espacio virtual de información, en el cual depende la capacidad crítica que pueda discernir de la veracidad de lo encontrado y actuar de manera reflexiva y creativa para generar un nuevo conocimiento.

Por otra parte, la reflexión de Fainhole (2003), se refiere al cuestionamiento sobre el reto de la educación tradicional no está en cohibir, restringir o castrar la oportunidad de vincular a los quehaceres pedagógicos las TIC, sino establecer procesos y estrategias frente a la información que hace parte de la sociedad globalizada. Por lo tanto, se debe abordar una metodología activa que propicie una postura crítica y reflexiva, alejando al estudiante de la práctica de copiar y pegar información.

Por otra parte, en Morales (2013), en la investigación titulada "Propuesta; un acercamiento a la lectura crítica para niños de grado cuarto por medio de herramientas TIC", encaminada a buscar lograr que los estudiantes se aproximen a la lectura crítica por medio de las TIC. Utilizó un libro de literatura infantil llamado Kaír Andrós, narrador de mitos del autor colombiano Andor Graut, en el cual se van mezclando dos formas de hacer lectura: la primera la tradicional, con libro en mano; y la segunda la digital, haciendo uso de presentaciones y juegos interactivos para probar cómo la tecnología aumenta la comprensión lectora de los estudiantes, integrando los procesos de lectura crítica a través de la adaptación de técnicas de comprensión
En la investigación de Fainholc (2006) en Argentina titulada "la lectura crítica en internet: desarrollo de habilidades y metodología para su práctica”, donde tuvo como objetivo establecer la relación entre el lector y la información digital en la generación de las competencias lectoras. 
propuestas por Daniel Casany. En esta experiencia se concluye que el uso de las TIC en la educación es un reto que debe ser asumido por los educadores, pues los estudiantes necesitan ampliar sus aprendizajes y tener una visión mucho más completa del mundo que los rodea. Tal como ella afirma: "No se trata de aceptar las tecnologías con los ojos cerrados, se trata de evaluar qué las herramientas son pertinentes y pueden ofrecer elementos formativos a los estudiantes”. (p. 91).

Por último, está la investigación por Hernández \& Arteaga (2011), titulada "Las TIC como facilitadoras en la comprensión lectora”. Resaltó ampliamente la importancia de establecer marcados procesos de inclusión en la comprensión lectora con las herramientas tecnológicas, articulando estrategias con diferentes características, lo cual no es una tarea fácil, pero si totalmente necesaria en la formación académica. La innovación tecnológica desde las aulas no radica meramente en la implementación de televisores, computadores o herramientas sistematizadas, sino en la destreza y estrategias que el docente plante y desarrolle para generar construcción de nuevo conocimiento y así propiciar espacios de análisis crítico, puesto que frente a un variado menú de información circulado es indispensable identificar el grado de relevancia de los diferentes contenidos a partir del desarrollo de competencias. Frente a la relación que establece la tecnología con el saber, fácilmente se toma el lenguaje como herramienta en escenarios de reflexión dado que por medio de éste los sujetos interactúan transformando los datos en conocimiento provisto de particularidades que llevan al lector no solo a saber del texto sino a investigar sobre el mismo.

\section{Referentes teóricos}

La investigación se fundamenta teóricamente desde tres ejes principales: la lectura crítica como herramienta para asumir posturas provistas de reflexión y opinión, las tecnologías de la información y comunicación, como los recursos mediáticos en el proceso enseñanza-aprendizaje y el uso de la estrategia pedagógica en el quehacer docente.

\section{La lectura critica}

En el proceso de la lectura Gordillo y Flores (2009), afirman que se comienza por la lectura literal, donde el lector reconoce la frase, las palabras clave, capta lo que dice sin intervenir en la estructura cognoscitiva, reconstruir el texto centrándose en las ideas o información explicita, por ejemplo: la idea principal. En este nivel también se puede llegar a identificar el orden de las acciones, de los lugares o de los tiempos. El siguiente nivel es el de lectura inferencial; se caracteriza por describir relaciones y asociaciones de significados que permiten al lector leer entre líneas, presuponer y deducir lo implícito, es decir, ir más allá de lo que se lee. Aquí se puede establecer una relación entre lo que se lee y los conocimientos con que ya se cuenta; infiriendo ideas principales, acciones, secuencias, relaciones e hipótesis, y por 
último el nivel crítico, en éste el lector no solo lee, también emite juicios sobre el texto, para aceptarlo o rechazarlo, pero con argumentos. El lector busca evaluar el texto, se permite leer detrás de líneas.

Durango Z. (2015), retomando las ideas de Gordillo y Flores (2009), afirman que la lectura literal como nivel básico centrado en las ideas, la información explicita en el texto y el reconocimiento de los detalles, como la identificación de secuencias de hechos y acciones. Luego sigue el nivel inferencial, donde se ubica la información implícita del texto, permitiendo comprender por medio de la relación y asociación el significado local y global del texto, para llegar a inferir hipótesis, y por último el nivel de lectura crítico, donde se realiza una comparación, asocia experiencias y opina a la luz de la reflexión personal del lector.

La lectura crítica se puede definir como una lectura analítica, donde se comprende lo que dice un texto; se analiza lo que puede llegar a expresar, verificando aciertos $\mathrm{y}$ errores en su modo de presentación. Ésta se da como un proceso de construcción de relaciones entre el autor y el lector, que hace necesario que este último posea algunos conocimientos previos para que logre contextualizar e inferir las intenciones del autor. Por esto en Cassany (2003) plantea que un lector crítico es aquel que:

(...) comprende autónomamente el propósito lingüístico, las intenciones pragmáticas y los puntos de vista particulares que subyacen a los discursos que le rodean; Toma conciencia del contexto (contenido cognitivo, género discursivo, comunidad de hablantes, etc.) desde el que se han elaborado dichos discursos; Puede construir discursos alternativos, que defiendan sus posiciones personales y que se vinculan polifónicamente o intertextualmente con los anteriores; Utiliza todos los recursos lingüísticos disponibles para conseguir representar discursivamente sus opiniones a través de esos segundos discursos. (pág. 114).

Al respecto se concluye que la lectura crítica es compleja, exige los más altos niveles de comprensión que sobrepasan lo literal y lo inferencial para provocar una postura externa del lector frente al texto, en el cual evidencie sus intenciones, su contenido y su punto de vista entre otros.

De otro lado Cassany (2003) enfatiza en la importancia que para un lector critico deben tener la connotación de las palabras y expresiones del discurso, para así poder valorar la idoneidad o efectividad de las mismas, delimitar la orientación argumentativa de cada parte del mismo, lo cual puede hacer que la lectura crítica sea una herramienta para obtener datos y reflexiones que lleven a generar hipótesis sobre el discurso, por ejemplo; a pensar en cuáles son sus causas, sus circunstancias, quién lo escribo, cuál es su sexo, edad, entorno social, sus ideas.

Así mismo la UNESCO (1988) citado por Serrano \& Madrid (2007), hacen alusión a la alfabetización crítica, de la siguiente manera: 
"alfabetización crítica pues está entraña el desarrollo de todas las capacidades básicas de comunicación que le permiten al hombre insertarse en el mundo del trabajo y en su cultura, como formas de realización personal y espiritual, de progreso social y desarrollo económico” (p. 54).

En atención a lo anteriormente expuesto se deduce que las múltiples ventajas de la lectura crítica para los estudiantes está en posibilitar la toma de una posición y conciencia frente a lo leído, que termina siendo una lectura reflexiva que tiene en cuenta el contexto del lector y del autor, propiciando que los lectores den un paso más frente a los procesos de enseñanza y aprendizaje de la lectura, pasando de leer las líneas y entrelíneas a leer detrás de la líneas, sin importar si el texto es físico o virtual, como lo propone Cassany, lo cual hace que el desarrollo de las habilidades en la lectura crítica favorezca el propósito lingüístico, las intenciones pragmáticas y los puntos de vista particulares que subyacen a los discursos que le rodean.

También es importante tener claro que la lectura crítica no necesita partir de un texto, también se puede realizar desde la lectura de un programa de televisión, de una imagen o de una historieta, por ejemplo, solo requiere una interpretación crítica de una realidad. O como lo resumen Cely A y Sierra E (2011):

"Hacer lectura crítica no es solamente entender el texto, tampoco es expresar sin ningún criterio una opinión. Si en cambio, es realizar de él una interpretación, fruto de la comprensión o estudio del texto. Es agregar significado, aplicándolo al mundo de la vida" en otras palabras los autores sostienen que "en la dimensión critica el elector identifica el significado que el texto encierra y la interrelación con los saberes previos que posee" . (pág. 17)

\section{Las Tecnologías de la información y comunicación en la educación}

El impacto de las TIC en todos los ámbitos de las sociedades del siglo XXI, ha influenciado cambios, substanciales en la educación, pues los aportes de las TIC como medio para el desarrollo de la propuesta educativa posibilitaron la transformación de nuevos escenarios y espacios pedagógicos institucionales, así como inesperados roles en los docentes y estudiantes.

El Ministerio de Educación Nacional (2008), para articular la tecnología en la educación introdujo este recurso a través del Plan Nacional de Tecnología de la información y comunicación, según el siguiente manifiesto:

... Diferentes organismos internacionales se han dado a la tarea de construir indicadores para evaluar en forma comparativa el desempeño de los países a nivel mundial y establecer las variables relevantes que determinan el grado de preparación para aprovechar los beneficios de las TIC. Lo cual establece la pertinencia de generar procesos de inclusión frente al manejo $y$ aplicación de las herramientas TIC en el ámbito académico y en general en los procesos de construcción y gene- 
ración de conocimiento desde y hacia las aulas de clase buscando elevar los niveles de desempeño y competencias de las futuras generaciones. (p.12)

Por otra parte, Colombia Digital (2013) en el desarrollo de su misión ha venido implementando estrategias para afianzar el uso de las TIC. Resalta al respecto las siguientes palabras:

"cómo el país, ha adoptado un marco jurídico, formulando políticas y acciones encaminadas a que éste transite hacia el fortalecimiento en la utilización de las TIC en las Instituciones Educativas que ayuden a la disminución de las actuales brechas de desarrollo social, resulta muy importante orientar las acciones a la promoción de estrategias que ayuden a la consolidación de este proceso y objetivo (p.32)

De acuerdo con Belloch (2012), afirmó que las Tecnologías de la Información y Comunicación en el aprendizaje "generaron un impacto sobre la educación, lo que propicio cambios en el ámbito de la Educación, el internet y sus múltiples recursos, permitió la posibilidad de acceder con facilidad a conocer personalidades de opiniones diversas" (p. 7).

De tal manera que progresivamente abrió nuevos espacios académicos de la mano con el desarrollo y evolución tecnológica, los cuales sin lugar a duda permitieron una fácil adaptabilidad no solamente de los escenarios educativos sino también los entornos sociales, los cuales ven en las herramientas tecnológicas, un avance significativo frente a la demanda de optimización de sus procesos productivos (Muñoz, 2016).
También, Marqués (2012) considera importantes los cambios que las tecnologías de la información y comunicación aportaron al ámbito educativo, expresando:

"La sociedad de la información incidió de manera significativa en todos los niveles del mundo educativo asimilando de forma natural esta nueva cultura que se va conformando y que para nosotros conlleva muchas veces importantes esfuerzos de formación, de adaptación y de "desaprender" muchas cosas que ahora "se hacen de otra forma" o que simplemente ya no sirven" (p.6).

Es así como los nuevos roles del docente en la sociedad de la información originaron la desinstalación del tradicionalismo, cambiando los paradigmas del protagonismo. De tal manera que el docente en el aula no es el actor de transmisión, sino aquel posibilitador de nuevos escenarios pedagógicos.

Desde esta perspectiva la comunicación y el conocimiento son dos interconexiones que proporcionan al estudiante el saber, el saber hacer, dejando atrás la rigidez para pasar a la potenciación de habilidades en el desenvolvimiento académico y el uso de las herramientas y medios que ofrece las tecnologías de la información y comunicación.

\section{Estrategia Didáctica}

La estrategia didáctica es la que permite el acoplamiento y aplicación de la pedagogía para la enseñanza, traducida “cómo el país, ha adoptado un marco jurídico, formulando políticas y acciones encaminadas a que éste transite bacia el fortalecimiento en la utilización de las TIC en las Instituciones Educativas que ayuden a la disminución de las actuales brechas de desarrollo social, resulta muy importante orientar las acciones a la promoción de estrategias que ayuden a la consolidación de este proceso y objetivo 


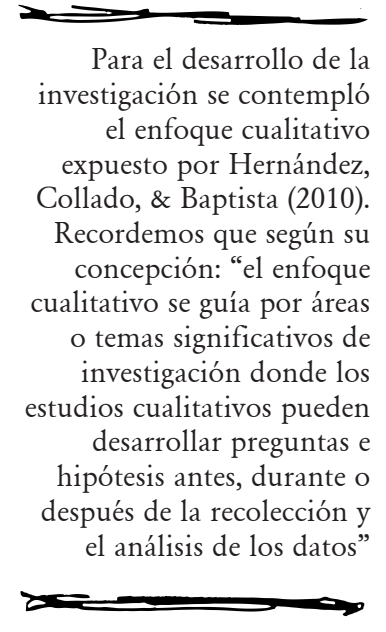

Para el desarrollo de la el enfoque cualitativo expuesto por Hernández,

Recordemos que según su concepción: "el enfoque cualitativo se guía por áreas o temas significativos de udios cualitativos pueden desarrollar preguntas e hipótesis antes, durante o el análisis recoleccion y en acciones planificadas por el docente con el objetivo que el estudiante logre la construcción del aprendizaje y alcance los propósitos planteados. Teniendo en cuenta lo afirmado por Díaz (2002) una estrategia didáctica "es un procedimiento organizado y sistemático que permite el desarrollo de competencias, mediante actividades que movilizan conocimientos, habilidades y actitudes, por tanto favorecen la apropiación o desarrollo de contenidos" lo que significa que a través de secuencias, procedimientos o planes orientados hacia la consecución de metas de aprendizaje, el docente construye y dinamiza el conocimiento y de ella depende que los estudiantes puedan y logren alcanzar y afianzar lo enseñado.

Por otra parte la estrategia didáctica es definida por Feo (2010) como los procedimientos, los cuales incluyen técnicas, actividades y métodos utilizados por los docentes y estudiantes, para lograr objetivos de enseñanza y aprendizaje, bajo una adaptación a la necesidades de los participantes en el proceso, y se pueden clasificar dichos procedimientos en estrategias de enseñanza, las cuales son personales entre docente y estudiante, estableciendo dialogo participativo, las instruccionales donde no es necesario establecer relaciones presenciales, pero existen por ejemplo materiales impresos que otorgan pasos a seguir, allí cobra importancia el uso de tecnologías como medio de difusión de dichas instrucciones, las de aprendizaje, que son las que realiza el estudiante conscientemente para aprender y que no necesitan del docente para llevarse a cabo, y las de evaluación donde se valorarán y describen los logros alcanzados por estudiante y docente, en atención a lo anterior el proyecto encaja ampliamente con una estrategia didáctica especialmente con las de enseñanza, las instruccionales y las de aprendizaje, donde se asegura una interacción entre docente, estudiante y medio tecnológico para lograr una meta, en el caso en particular de reforzar lectura crítica, a través de una página web.

\section{Metodología}

Para el desarrollo de la investigación se contempló el enfoque cualitativo expuesto por Hernández, Collado, \& Baptista (2010). Recordemos que según su concepción: "el enfoque cualitativo se guía por áreas o temas significativos de investigación donde los estudios cualitativos pueden desarrollar preguntas e hipótesis antes, durante o después de la recolección y el análisis de los datos"(p.4), donde se plantea un problema, y se va de lo particular a lo general, no se prueban hipótesis, estas se generan durante el proceso de indagación que es flexible y evalúa el desarrollo natural de los sucesos, introduciendo las experiencias de los participantes, y el proceso se adelantará bajo la investigación acción-educativa, donde Elliot (2000) plantea que: La investigación-acción educativa se relaciona con los problemas prácticos cotidianos experimentados por los profesores, y que su propósito consiste en profundizar la comprensión de su problema. 
La metodología de la presente investigación toma como referencia, el proceso planteado por Elliot, para lo cual se tomaron y adaptaron tres etapas:

1. Etapa de planificación: Busca identificar un problema a partir de un interrogante, reconocerlo y realizar un diagnóstico. Teniendo en cuenta el contexto, esto se pretende lograr a través de las siguientes actividades:

- Observación directa: observar a las estudiantes involucradas en el proceso para caracterizar $\mathrm{y}$ conocer sus niveles de lectura crítica e implementar las técnicas de observación directa orientadas hacia cómo se utilizan los recursos tecnológicos frente a la lectura y registrar aquellos hechos que son susceptibles de ser interpretados.

- Revisión documental: realizar revisión de documentos como el PEI, el plan de mejoramiento institucional y los resultados del ISCE, para detectar las necesidades más apremiantes de la institución, en relación a la lectura crítica y el manejo adecuado de herramientas TIC.

- Caracterización: Realizar la recolección de información a través de un formulario, cuyo propósito es identificar habilidades críticas, fortalezas y debilidades frente al manejo de la lectura crítica, en pruebas internas y externas.

2. Etapa de acción: Partiendo de los hallazgos que se encontraron en la primera etapa, se establecen las siguientes actividades:

- Construcción de la estrategia didáctica: se buscó la mejor alternativa para lograr interactividad entre las estudiantes y los procesos de lectura crítica.

- Desarrollo: consistirá en crear el espacio Web, y luego hacer que las estudiantes del grado décimo en las áreas de Física y Sociales iniciaran un proceso de interacción y socialización de diferentes temáticas, haciendo publicaciones y desarrollo de actividades.

3. Observación y reflexión: En esta etapa se analizan y describen todos los elementos encontrados a partir del desarrollo de la estrategia didáctica, donde se encontrarán elementos que soporten si fue o no importante su aplicación y el impacto de las diferentes actividades en las estudiantes, manteniendo siempre un abordaje crítico, elaborando el informe y por último replanteando o aseverando el problema teniendo en cuenta que es, o no, una teoría válida, si se puede llevar a la práctica y triangulando la información con los referentes teóricos hallando las conclusiones del estudio

Por otro lado, refiriendo la población de la investigación, en este caso el Colegio Nacionalizado la Presentación del municipio de Duitama - Boyacá, de carácter oficial, femenino, el cual cuenta con 2.350 estudiantes entre preescolar, primaria, secundaria y media, pertenecientes a estratos 1, 2 y 3. Cuya muestra se seleccionó 
intencionalmente; el grado décimo, tomando un total de 35 estudiantes.

De otro lado y en atención al contexto de investigación, se contemplaron los aspectos correspondientes a las consideraciones éticas relacionadas con el desarrollo de la investigación referida.

\section{Resultados y análisis}

Los resultados presentados corresponden al desarrollo total de la etapa uno y parte de la etapa dos, la investigación se encuentra en curso.

Etapa de planificación: Buscaidentificar y reconocer el problema a partir de un diagnóstico, teniendo en cuenta el contexto. Esto se pretende lograr a partir de las siguientes actividades: primero se realizó un diagnóstico que comenzó con la observación directa de las clases de Física y Sociales, proponiendo pequeños textos para ser leídos y analizados donde se pudo evidenciar la falta de habilidades e interés por la lectura crítica, por parte de las estudiantes de grado décimo, ya que ellas prefieren actividades que no involucren lectura de textos y por el contrario donde la tecnología tenga gran participación, dichos hallazgos se encuentran consignados en el diario de campo.

Por lo anterior, se establecen estrategias de mejoramiento no solo identificar falencias y posibles planes de acción sino diseñar o rediseñar tal y como lo argumenta Díaz (2002), "procedimientos organizados y sistemáticos que permitan la movilización de conocimientos, habilidades y actitudes", para propiciar escenarios que despierten curiosidad por la investigación, experimentación y abrir espacios prácticos en la nueva forma de asumir cambios frente a la dinámica de la sociedad del conocimiento del siglo XXI.

Por otra parte, Belloch (2012) afirma que las Tecnologías de la Información y Comunicación en el aprendizaje "generó un impacto sobre la educación, lo que propicio cambios con el uso de la internet y sus múltiples recursos”, permitiendo conocer diversas opiniones producto de posiciones frente a situaciones cotidianas, científicas $\mathrm{y}$ de diferentes temas, lo que también apoyo a gestionar competencias en todos los niveles de formación docente y estudiantil, de tal manera que la adaptabilidad a las nuevas herramientas movilizara el conocimiento en provecho del crecimiento humano en todos sus contextos que para Marqués (2012), lo considero como la "sociedad de la información" influyendo de manera significativa el "desaprender" muchas cosas que ahora "se hacen de otra forma" o que simplemente ya no sirven, conllevando a la generación de una transformación de métodos, técnicas y uso de instrumentos para lograr escenarios propicios y favorables en la enseñanza-aprendizaje con base en nuevos referentes para integrar en los sistemas educativos como respuesta a los progresos de la tecnología y la dinamización de la educación.

En este sentido, las tecnologías juegan un papel de vital importancia, al brindar 
una integración entre la adquisición, la destreza, el reconocimiento, el manejo de los medios tecnológicos, y la forma como estos presentan la oportunidad de abordar contenidos textuales, informativos, imágenes, entre otros; pretendiendo romper los esquemas del tradicionalismo pedagógico.

Posteriormente para continuar con las actividades de la etapa de planificación se acude a una revisión documental frente a los resultados obtenidos en el ISCE, en el cual se encuentra un comparativo del promedio institucional alcanzado en relación a los años posteriores, reflejando un aumento en los índices de lectura crítica, dejando ver que la implementación de una estrategia didáctica contribuiría a superar estos resultados, y dado que el decreto 1075 del 2015 relaciona el índice de calidad como herramienta de medición y que está conformado por el índice de calidad educativa (ISCE) y el índice de gestión de calidad educativa (IGCE); hace de este documento vital para encontrar las necesidades de la institución.

Por otra parte también se tuvieron en cuenta para su análisis los resultados obtenidos en pruebas de estado 2016 a nivel institucional los cueles reflejan un avance en comparación con años anteriores, lográndose evidenciar que la búsqueda e implementación de nuevos espacios de motivación y generación de habilidades en lectura crítica contribuirían a la obtención de mejores resultados a nivel general teniendo presente que la lectura crítica desempeña un papel relevante en cada una de las competencias que hacen parte de estas pruebas.

La estrategia propuesta en este texto proporcionará los medios necesarios para elevar los valores de Aceptable en lo referente a la lectura crítica que a pesar de las pruebas de estado en la institución y de su progresivo ascenso en tres años requiere una especial atención para fortalecer las debilidades y permita el desarrollo de habilidades en lectura crítica, competencia que implica tal y como lo fundamentan Gordillo y Flores (2009), pasar por etapas como la lectura literal, donde se reconocen la idea principal y lo explícito del texto, la lectura inferencial, donde se construye hipótesis y se deduce, para luego pasar al nivel crítico, donde se analiza, interpreta, compara, valora y opina, todo estos procesos conllevan a desarrollar capacidades para abordar una lectura profunda que pueda generar en el lector emitir juicios sobre el texto, para aceptarlo o rechazarlo argumentando de manera coherente su punto de vista. En este sentido Cely y Sierra (2011) se refieren a que "no es solamente entender el texto, tampoco es expresar sin ningún criterio una opinión, sino realizar del texto una interpretación, fruto de la comprensión" o "agregar significado, aplicándolo al mundo de la vida" lo que requiere la interrelación de saberes previos y asociar significados sometidos a un anclaje que encierra de forma particular las ideas y posiciones del lector.

Para continuar con la actividad de caracterización propuesta en la etapa
Cely A y Sierra E (2011) se refieren a que "no es solamente entender el texto, tampoco es expresar sin ningún criterio una opinión, sino realizar del texto una interpretación, fruto de la comprensión" o "agregar significado, aplicándolo al mundo de la vida” 
En el colegio Nacionalizado la Presentación se realizan tres pruebas externas de este tipo, de las cuales se utilizó

la que se presentó en el segundo semestre de 2016

EdUCACIÓN Y CienCIA - NÚM 22 . AÑo 2019 • PÁG. 263-277 de planificación se tuvieron en cuenta las pruebas externas que la institución ofrece periódicamente en alianza con el grupo Instruimos; empresa que se dedica a realizar pruebas en diferentes establecimientos educativos con el ánimo de identificar fortalezas y debilidades frente a los procesos académicos que se llevan a cabo en las instituciones del país. En el colegio Nacionalizado la Presentación se realizan tres pruebas externas de este tipo, de las cuales se utilizó la que se presentó en el segundo semestre de 2016, para conocer los niveles de lectura crítica de las estudiantes de la muestra, los resultados arrojados en esta prueba reflejan la necesidad de un plan de mejoramiento ya que se cuenta con un grupo en nivel bajo y la gran mayoría de las estudiantes en nivel medio, lo que permitió identificar la oportunidad de mejoramiento en dicho campo académico, a través de la creación e implementación de una estrategia didáctica que busque mejorar los niveles de lectura crítica en las estudiantes.

Por otra parte, otra actividad de caracterización contemplada en la primera etapa de la investigación, se realizó una prueba interna donde las estudiantes del grado 1004 tuvieron la oportunidad de demostrar que son un curso que cuenta con habilidades críticas básicas para su clasificación y valoración. Se recurrió a la implementación del formulario para determinar las habilidades críticas que se adaptó del curso en línea Pensamiento Crítico, dirigido por el RIED (Red Interamericana de Educación Docente), y permitió clasificar e identificar que un gran número de estudiantes ya tienen un proceso de desarrollo en estás habilidades. De igual manera aproximadamente la mitad de las estudiantes evidenciaron en el ítem de lectura crítica una valoración de aceptable y la otra mitad del grupo un alto grado. Lo que suscitó focalizar la oportunidad de mejoramiento a partir de la necesidad de trabajar sobre las debilidades que presentaron los estudiantes en el ítem de lectura crítica a través de la creación de una estrategia mediada por las tecnologías de la información y comunicación.

Por lo tanto, el desarrollo de habilidades para lograr un desenvolvimiento favorable en el proceso de la lectura crítica requiere primero potenciar habilidades inferenciales, para luego posibilitar el análisis e interpretación permitiendo según, Durango (2015), "comprende por medio de relaciones y asociación el significado local y global del texto, logrando explicar las ideas más allá de lo manifestado explícitamente en el texto, sumando información y experiencias anteriores para llegar a la elaboración de conclusiones" producto de esta comprensión y agregando un nuevo sentido originado del punto de vista propio, auténtico y proporcional a lo expuesto del tema central de la lectura que evidencie, según Cassany (2003) "el propósito lingüístico, las intenciones pragmáticas y los puntos de vista particulares que subyacen a los discursos que le rodean", lo que indica que el lector ha tomado y construido con los recursos lingüísticos disponibles expresar su opinión producto de la 
movilización del conocimiento y la información que se articuló en el texto.

Para continuar las TIC, se evidencia que son herramientas propicias para el desarrollo de las capacidades en lectura crítica y el diseño de la estrategia está enlazada y adaptada para que los estudiantes experimenten a través de las actividades las ventajas y aprovechamiento de estos espacios para interactuar y dinamizar los saberes, las opiniones y la manipulación responsable de la información, pues una de las características particulares del ciberespacio es la disponibilidad de recursos permanentes, lo que permitirá una interacción continua en las actividades propuestas presentadas y diseñadas con el propósito mejorar los procesos de enseñanza-aprendizaje centradas en la lectura crítica.

En la segunda etapa llamada de acción se analizaron los hallazgos que se encontraron en la primera etapa y se empezó a construir la estrategia didáctica planteada. Inicialmente estableciendo en conjunto con las estudiantes, los criterios y elementos que sirvieron para fundamentar $y$ enriquecer la propuesta, mediada a través de herramientas TIC, con la creación de una página web que asegure la motivación y al mismo tiempo la lectura crítica usando imágenes, artículos y otros; contribuyendo a potenciar o desarrollar las habilidades para la misma y para dinamizar la interacción y el desenvolvimiento progresivo de la capacidades de la comunicación, y con ellas las habilidades críticas de las estudiantes.
Desde la mirada de la alfabetización crítica según UNESCO (1988) citado por Serrano y Madrid (2007), se requiere "el desarrollo de capacidades básicas de la comunicación" indispensables para el desenvolvimiento efectivo en escenarios futuros donde el ser humano atienda las demandas y dé respuesta apropiada a el mundo laboral, profesional, social, económico $y$ personal en diferentes momentos donde se solicite una posición crítica, oportuna y razonable que esté acorde en los diferentes contextos donde se movilice el hombre.

Por otra parte, y para continuar con las actividades de la segunda etapa, se realizó una adaptación de las técnicas de lectura crítica de Cassany (2003), donde las estudiantes aportaron de manera significativa a la elección de las técnicas que se utilizaran, en la actualidad se están realizando actividades con cuentos y caricaturas para buscar que las estudiantes del grado décimo en las áreas de Física y Sociales interactúen, generen y socialicen diferentes temáticas que permitan explorar a las estudiantes sus habilidades en lectura crítica, utilizando las técnicas propuestas por Daniel Cassany, y que se adaptaron para el grupo de estudio.

\section{Conclusiones}

La lectura crítica implica un proceso de aprehensión de información que subyacen dentro de un texto escrito evidenciando una movilización de lectura analítica, reflexiva y activa; producto de la comprensión 
profunda de un texto en su totalidad, desentramando el mensaje implícito del contenido más allá de lo literal, que hace posible evaluar y formar un juicio con fundamento.

En la estrategia para el mejoramiento de la lectura crítica en los estudiantes de décimo grado se articuló el uso de las tecnologías de la información y comunicación para aprovisionar al educando de herramientas actuales en su aprovechamiento y manipulación, lo que permitió promover escenarios favorables para la interacción $y$ despliegue de diversos formatos que invitan al estudiante a desarrollar una lectura dinámica dotada de aplicaciones y diseñada con base en condiciones específicas necesarias para el desarrollo de habilidades y capacidades focalizadas en el análisis, reflexión y opinión.

Losdocentes porsu parteexperimentaron nuevos retos que proporcionaron a la práctica pedagógica el enriquecimiento de la planificación sistemática y auténtica empleando las tecnologías de la información y comunicación como una aliada recursiva en la metodología para favorecer los saberes y enseñanza en la lectura crítica mediante actividades innovadoras que permitieron contribuir al desarrollo del proyecto.

\section{Bibliografía}

Belloch, C. (2012). Las Tecnologías de la información y comunicación (T.I.C.) en el aprendizaje. España: Unidad de Tecnología Educativa. Universidad de Valencia.

Cassany, D. (2003). Aproximaciones a la lectura crítica: teoría, ejemplos y reflexiones, Revista Taibiya, revista de investigación e innovación educativa, 32 Disponible en https://revistas.uam.es/tarbiya/article/viewFile/7275/7623

Durango, Z. (2015). La lectura y sus tipos. Portal de las Palabras. Revista virtual de Proyecto Institucional de competencias comunicativas. Corporación Universitaria Rafael Núñez.

Elliott, J. (2000). La investigación - acción en educación. España: Morata, S.L.

Fainholc, B. (2006). Investigación: La lectura crítica en Internet, Desarrollo de habilidades y metodología para su práctica. AIESAD. Disponible en; http:// www.biblioteca.org.ar/libros/142141.pdf.

Feo, R. (2010). Orientaciones básicas para el diseño de estrategias didácticas. Revista Tendencias Pedagógicas, 16, Disponible en; http:// https://repositorio. uam.es/bitstream/handle/10486/5273/33795_2010_16_13.pdf 
Hernández, J., \& Arteaga, D. (2011). Las TIC como facilitadoras en la comprensión lectora. (Tesis de especialización) Universidad Católica de Pereira, PereiraColombia.

Hernández, R., Fernández, C., \& Baptista, P. (2010). Metodología de la Investigación.

México: Mc Graw Hill.

Latorre, A (2003). La investigación - acción conocer y cambiar la práctica educativa. España: Graò S.L.

Ministerio de Comunicaciones (2008), Plan Nacional de Tecnologías de la Información y las Comunicaciones, 2008-2019, Colombia

Morales, L. (2013). Un acercamiento a la lectura crítica para niños de grado cuarto por medio de Herramientas TIC. (Tesis de pregrado) Universidad Libre, BogotáColombia.

Muñoz, H. (2016). Mediaciones tecnológicas: nuevos escenarios de la práctica pedagógica. Praxis \& Saber, 7(13), 199 -221. https://doi. org/10.19053/22160159.4172

Ortiz, O. (2006, marzo). Lectura y escritura en la era digital. Edutec Revista electrónica de tecnología Educativa, 17.

Serrano S., \& Madrid A. (2007). Competencias de lectura crítica. Una propuesta para la reflexión... Revista Acción Pedagógica, (16), 58 - 68. 